\title{
RESUMEN.
}

El presente documento tiene como finalidad analizar las distintas teorías que intervienen en la cooperación empresarial. El análisis que se pretende realizar tiene como propósito establecer una línea de tiempo, que a través de los años ha generado alguna aportación para el estudio y desarrollo de la cooperación empresarial, debido a que se espera aportar una simplificación de la relación y comparación evolutiva para poder comprender desde una perspectiva diferente las temáticas tratadas para el alcance de este término.

Palabras Clave: Cooperación empresarial, Teoría organizativa, Innovación.

\section{IMPLICACIONES DE LAS TEORÍAS ORGANIZATIVAS EN LA CONSTRUCCIÓN DE LA COOPERACIÓN EMPRESARIAL}

\author{
Jovanni Trinidad Saldaña / José Sánchez-Gutiérrez / Katia Magdalena Lozano Uvario
}

\section{Fecha de recepción: 14 de junio de 2019 \\ Fecha de aceptación: 11 de diciembre de 2019}

\section{DOI: https://doi.org/10.22370/riace.2019.8.1.2070}

\section{Jovanni Trinidad Saldaña}

Doctorado en Ciencias de la Administración, Centro Universitario de Ciencias Económico Administrativas, Universidad de Guadalajara.

Calle: Periférico Norte $N^{\circ} 799$, Núcleo Universitario de los Belenes, ZIP 45100, Ciudad: Zapopan, Estado: Jalisco, México.

José Sánchez Gutiérrez

Departamento de Mercadotecnia y Negocios Internacionales, Centro Universitario de Ciencias Económico Administrativas, Universidad de Guadalajara.

Calle: Periférico Norte № 799 , Núcleo Universitario de los Belenes, ZIP 45100, Ciudad: Zapopan, Estado: Jalisco, México.

Katia Magdalena Lozano Uvario

Departamento de Geografía y Ordenación Territorial, Centro Universitario de Ciencias Sociales y Humanidades, Universidad de Guadalajara.

Calle: José Parres Arias №150, Núcleo Universitario de los Belenes, ZIP 45100, Ciudad: Zapopan, Estado: Jalisco, México. 


\section{THEORETICAL IMPLICATIONS IN BUSINESS COOPERATION}

\section{ABSTRACT}

The purpose of this document is to have an analysis of the different theories that intervene in business cooperation and innovation. The purpose of the analysis is to establish a timeline of the different theories established and that over time have generated some contribution for the study and development of the business cooperation, due It is expected to provide a simplification of the relationship and evolutionary comparison to understand from a different perspective the issues addressed.

Keywords: Business cooperation, Organizational theory, Innovation. 


\section{INTRODUCCIÓN}

La cooperación empresarial, así como las teorías organizativas, tienen desde principios del siglo XIX teniendo una gran interacción entre ambas con el objetivo de plantear acciones que lleven a las organizaciones a ser más competitivas frente a las problemáticas que las grandes empresas vienen a generar en los sectores más vulnerables de las economías de escala, es por ello, que se alcanza a visualizar como la cooperación entre los mismos empresarios y sus intentos día con día por introducirse dentro del ámbito empresarial ha llevado a la implicación de varias acciones argumentadas tanto por Alfred Marshall en su obra literaria de los Principios de economía sobre la importancia de los valores dentro de las empresas u organizaciones para establecer vínculos cooperativos dentro de las acciones que lleven a la interrelación empresarial con el objetivo de promover la creación de valor (Marshall, 1930).

Transcendiendo la cooperación empresarial hasta los años 70 , es que fue implementada por las grandes empresas multinacionales, con la idea de lograr conseguir apoyo a través de pequeñas empresas que fungieron como socios locales de cada uno de los países donde decidieron incursionar, lo que permitió generar un elevado crecimiento de los negocios cooperativos y las sociedades mercantiles. A partir de los años 80 se encuentra un detonante sobre las grandes aportaciones de las acciones de cooperación empresarial (Urra, 1999), ya que, es uno de los momentos donde las grandes teorías organizativas comienzan a argumentar situaciones que motivaban a los empresarios a participar en conjunto, además, se puede referir que en estos años la participación de las empresas tuvo un incremento considerable sobre de los acuerdos de cooperación empresarial.

En 1990 se generó un fuerte cambio organizacional en los negocios, por tal motivo, las corporaciones decidieron llevar a cabo la reorganización de su estructura, formando en las mismas un enfoque descentralizado, menos jerárquico y dirigido a actuar como pequeñas y medianas empresas, donde se comprendieron múltiples asociaciones (Urra, 1999). Para Cabanelas y Sáenz (1997) supone que la cooperación corresponda a la presencia de "nuevos" factores, tanto en el ámbito externo considerando el entorno que les acontece, como en el interno de las empresas que están provocando cambios sustanciales en la forma de competir. Por otra parte, Cabanelas y Sáenz (1997) argumentan que la cooperación empresarial es él establecer una relación "voluntaria y recíprocamente el compartir algún recurso y/o conocimiento de tipo tecnológico, comercial o financiero con el objeto de desarrollar una estrategia que redunda en ventajas competitivas para los cooperadores" en otras palabras, la cooperación implica considerar a Ios proveedores, clientes y competidores como posibles aliados y como fuente de información, lo que promueve en un momento determinado un ambiente de mercado concertado.

Dentro del siglo XX inicia el fomento formal de los acuerdos cooperativos por parte de los empresarios, además, de las colaboraciones en innovación y desarrollo 
como medio eficaz para las empresas de cara a lograr nuevos y mejores productos y procesos, de acuerdo a lo mencionado por el Instituto Andaluz de Tecnología. A su vez, dentro del ámbito internacional durante la conferencia en Estambul, en el 2004, los representantes de los países miembros de la Organización para la Cooperación y el Desarrollo Económicos (OCDE), reconocieron que la PYMES juega un papel clave en el crecimiento económico sostenido y en la creación de empleos; que ésta contribuye al desarrollo social, cultural y económico de las naciones.

Reafirmaron durante dicho encuentro, su compromiso para trabajar en establecer políticas que respalden el desarrollo, crecimiento y competitividad de las PYMES; para buscar el diálogo político y la cooperación respecto a este asunto con los países miembros de la OCDE y con los no miembros. Esto implica a la PYME como motor de desarrollo, focalizando los esfuerzos no sólo a la creación y desarrollo de empresas sino también a la internacionalización de las mismas; sin que éstas pierdan su referéndum nacional, para con esto último, mantener el desarrollo local, por lo que la creación de redes de cooperación empresarial internacionales puede ser la respuesta a esto.

En la Comisión Europea en el 2015 tomó la decisión de incrementa la inversión y la cooperación empresarial con América Latina y el Caribe, por parte del Comisario Mímica, responsable de Cooperación Internacional y Desarrollo, ha declarado Io siguiente: "La Cumbre que celebramos hoy es una oportunidad para que las empresas de ambos lados del Atlántico se reúnan, compartan ideas y refuercen sus vínculos. Los nuevos programas que se han firmado hoy contribuirán a reforzar aún más el crecimiento integrador para las sociedades de Europa y América Latina.»

La estrategia empresarial conocida como cooperación empresarial en la actualidad, ha sido utilizada exitosamente en diversas regiones del mundo y bajo condiciones socio-geográficas y económicas muy diferentes de acuerdo al contexto del país 0 región donde esta se esté realizando, tomando en cuenta como sus principales acciones en los países tales como: Alemania, Italia, Francia, España. Estados Unidos, quien puede ser el ejemplo más claro de éxito empresarial a través de aglomeraciones industriales, se considera ya que en estos momento cuenta con varias ciudades que favorecen este tipo de práctica empresarial para atraer nuevas industrias hacia ellos y desarrollar tecnológicamente su región.

\section{REVISIÓN DE LITERATURA}

La revisión literaria conlleva la búsqueda de los distintos enfoques teóricos y las aproximaciones que han tratado de explicar la decisión de forjar la cooperación empresarial como estrategia competitiva, por otra parte, se produjo la investigación de los antecedentes que llevaron a profundizar en los distintos autores y sus estudios que permitieron asimilar la relación para la simplificación y comparación evolutiva de las distintas perspectivas teóricas. 


\section{IMPLICACIONES DE LAS TEÓRICAS ORGANIZATIVAS EN LA COOPERACIÓN EMPRESARIAL}

Las organizaciones en la actualidad se encuentran presentes en todos lados, los cambios constantes en su entorno las han llevado a desarrollar distintas actividades para hacer frente a sus necesidades básicas. Dentro de las estructuras organizacionales es importante comprender sus elementos y fundamentos que las conforman, pero también, es relevante vislumbrar aquellas similitudes que pueden apoyar en la generación de estructuras organizacionales, por lo que, un sustento teórico abona en la comprensión de las empresas, así como el desarrollo de acciones y estrategias como lo es la cooperación empresarial.

Con respecto a lo anterior mencionado, se llevó a cabo la investigación de las distintas teorías organizacionales y sus aportaciones hacia la importancia y relevancia de la cooperación empresarial, debido, a que el correcto análisis de estas teorías puede generar una aportación en la interpretación de la importancia de esta estrategia y como la consideran desde múltiples perspectivas en el desarrollo de las organizaciones.

\section{Teoría Neoinstitucional y Relaciones Interorganizacionales}

La teoría neoinstitucional propuesta por Powell y DiMaggio (1983), se concentró junto con las relaciones interorganizacionales considerándolas como parte de una misma acción, por lo que se tomó en cuenta para nombrarlas por su similitud y características principales con respecto a la teoría de la organización, que genera interactividad y adaptabilidad para cubrir la necesidad principal, sobrevivir y adaptarse buscando la legitimidad (Bejarano Lugo, 2016).

Para Powell y DiMaggio (1983) el proceso es una "estructuración" y consta de cuatro partes (1) el crecimiento de la interacción entre las organizaciones en el campo, (2) emergencia entre sus patrones de coalición y estructuras de dominación, (3) un incremento en la carga de información en las cuales las organizaciones tienen que enfrentarse y por último (4) el desarrollo del conocimiento recíproco entre los participantes de un conglomerado de organizaciones que están relacionados en una empresa en común (Bejarano Lugo, 2016).

Por otra parte, es importante mencionar que la teoría interorganizacionales abordada por Evan (1967) indicaba que las relaciones e interacciones con otras organizaciones, se daban a partir de las constantes interacciones hacia una búsqueda de legitimidad, aceptación social, adaptación organizativa, ambientes turbulentos y cambiantes así como a la normatividad impuestas entre organizaciones (Bejarano Lugo, 2016).

\section{Teoría del Caos, Anarquías Organizadas}

La teoría del caos tiene su origen dentro del campo de la física y las matemáticas, donde a partir del hallazgo de fenómenos dinámicos no lineales cuyas acciones 
parecían aleatorias, aun cuando se realizaban determinadas por leyes precisas, se volvían sistemas dinámicos no lineales que se comportan de manera impredecible y caótica (Pidal Gonzalez, 2009).

Con respecto a lo anterior, Dolán García y Auerbach (2003) mencionan que los fenómenos dinámicos de la teoría del caos es posible aplicarlos dentro de la gestión de las organizaciones a partir de comprender la relación entre el orden y el desorden. Por tal motivo en el contexto de la cooperación empresarial nos ayuda para considerar la toma de decisiones dentro de un orden, que lleve a la interacción de las organizaciones a generar una sinergia positiva a través de la comprensión de los sistemas dinámicos y los alcances que se pueden tener dentro de los acuerdos mutuos.

\section{Teoría de los costes de transacción}

La Teoría de los Costes de Transacción fue desarrollada por Hebert Simon (1937) y ha sido propuesta por numerosos autores como un modelo teórico explicativo de los acuerdos de cooperación (Kogut, 1988). Esta teoría, menciona que la cooperación, en algunas ocasiones puede llegar a determinar la aplicación de los recursos de manera más eficiente que el mercado y la empresa. Así, la cooperación funge en la relación entre el mercado y la organización como una intermediación, que en ciertos momentos, puede llegar a corregir imperfecciones que se presentan y a enfrentar los posibles cambios en su entorno (Williamson, 1979; 1985; 1991).

La cooperación se puede considerar como una forma de realizar intermediación entre la empresa y el mercado, lo que permite que se establezca como una nueva opción para la obtención de recursos. A partir de ella, las acciones que se generan dentro de las relaciones y en la toma de las decisiones no se fundamentan en la jerarquía ni tampoco en el mercado, sin embargo, se realizan a partir de las reglas internas acordadas previamente negociadas (Williamson, 1979).

La cooperación puede obtener un mayor grado de eficiencia siempre que las empresas que cooperen consigan costes más bajos en una manera integrada, sin embargo, esto sólo puede ser sí los costes de transacción de la cooperación son más bajos que los causados por las relaciones de mercado (Luo, 2002); (White \& Siu-Yun, 2005). Considerando los costes, será necesario que la empresa decida en todo momento sobre la mejor opción a elegir, ya sea: mercado, internalización 0 acuerdo de cooperación. Cabe mencionar que un análisis coste-beneficio de los casos indicará cual es la mejor alternativa (Williamson, 1991; Casani, 1996).

Kogut (1988) y García Canal (1996) a partir de la aplicación de esta teoría intentan explicar cómo los acuerdos de cooperación pueden desarrollar soluciones eficaces en los problemas relacionados con la incertidumbre de la conducta en los socios y en los oportunistas, a través de la obtención de una alianza, de la cual se permite posicionar la relación en "mutua dependencia". Es importante mencionar que dependiendo de la complejidad en los acuerdos con los que se puede incrementar los costes de transacción exponencialmente. 
Para finalizar, se puede asegurar que la Teoría de los Costes de Transacción argumenta que la misma es una base teórica primordial en la cooperación, debido a que explica las ventajas que puede contribuir frente al mercado o la empresa, además puede analizar las condiciones económicas la alianza, partiendo de que se considera como un mecanismo eficaz para la asignación de recursos en comparativa con algunos otros.

\section{Teoría de la gestión del conocimiento}

La Teoría de la gestión del conocimiento va dirigida a la generación organizacional que pretendía efectuar la innovación en procesos, productos y funciones de las organizaciones. Se considera que la innovación es una característica de valor agregado que se genera a partir de obtener un cambio y/o gestionar el conocimiento. Nonaka y Takeuchi (1995), crearon un modelo cuya finalidad fue gestionar el conocimiento, a partir de la cual describieron la relevancia del conocimiento explícito y el tácito, además de cómo reaccionan a partir de una interacción social para utilizarlo en la generación de conocimiento favoreciendo a la organización empresarial.

\section{Teoría de la dependencia de recursos}

La teoría de la dependencia de recursos (Aldrich, 1976; Aldrich \& Pfeffer, 1976; Pfeffer \& Salancik, 1978), la cual se refiere a la escasez de los recursos en la interdependencia entre organizaciones.

Dentro de la Teoría de Dependencia de Recursos se cree, que las organizaciones desarrollan un crecimiento en las interacciones empresariales con la finalidad de disminuir la incertidumbre en su entorno y se generan los acuerdos cooperativos. Así mismo, Escribá y Menguzzato (1999) señalan que la dependencia entre los socios de una cooperación será determinada a través de la relevancia dada a los acuerdos estratégicos y económicos de las empresas.

A su vez, Emerson (1962) argumenta, que considerando el nivel de dependencia en una relación, se puede decir que se vuelve recíprocamente proporcional a la probabilidad de que una empresa pueda llegar a sus metas al finalizar dicha relación.

Por otra parte, Pfeffer y Salancik (1978) dicen que las alianzas pueden lograr ser más fuertes siempre y cuando las mimas tengan menor dependencia, debido a que esto no permitirá tener alguna posibilidad en el comportamiento de los otros participantes, sino sólo lo necesario para el cumplimiento de sus propios objetivos, sin embargo es importante mencionar que cada participante genera un beneficio mutuo en la interrelación, por lo que la aportación en las empresa se genera por el comportamiento del socio (Rodríguez \& Wilson, 2002).

Según Gulati y Westphal (1999) las alianzas que en algunas ocasiones son utilizadas por las organizaciones, lo hacen delimitando la dependencia hacia otras 
empresas inmersas en el mercado, con la finalidad de garantizar el ingreso de futuros recursos que difícilmente poseerían individualmente. A su vez, Westphal, Boivie y Han (2006) suponen que si las organizaciones inician con relaciones formales las empresas podrán cooperar en la adquisición de conocimientos y habilidades que disminuyan el grado de dependencia, sin embargo, en el momento en que los participantes alcancen sus objetivos es posible que la cooperación finalice (Inkpen \& Beamish, 1997).

\section{Teoría de recursos y capacidades}

Según Gulati y Westphal (1999) las empresas pueden usar las alianzas para limitar la dependencia que tienen de otras entidades en el mercado, y de este modo tratar de asegurarse el acceso futuro a flujos de recursos que ellas no poseen de forma aislada. Westphal, Boivie y Han (2006) consideran que en las relaciones formales entre organizaciones, como las desarrolladas en los Consejos de Administración, se gestiona la dependencia de recursos y se pueden establecer futuras alianzas con el objeto de aprovechar la complementariedad de sus activos y reducir la incertidumbre asociada al acceso a los recursos que se necesitan. Sin embargo, si las empresas cooperan para adquirir conocimiento y habilidades que reduzcan su nivel de dependencia, cuando alguno de los socios cumpla sus objetivos existen muchas posibilidades de que la cooperación finalice (Inkpen \& Beamish, 1997).

Dentro de la teoría de recursos y capacidades la cooperación nace con la necesidad de encontrar un camino hacia la obtención de recursos que la empresa requiere y no ha podido conseguir (Das \& Teng, 2000), o como un camino para mejorar y disponer de los recursos a partir de la acuerdos alcanzados con los socios para conservar la ventaja competitiva (Eisenhardt \& Scoonhoven, 1996).

En conclusión, la Teoría de los Recursos y Capacidades se afirma como una de las teorías explicativas fundamentales que establece tanto la formación como la gestión de los acuerdos de cooperación.

\section{Teoría del desarrollo endógeno}

La teoría del desarrollo endógeno fue fundamentada a partir de que las economías de las regiones y localidades se creen con probabilidad de crecimiento considerando el potencial de progreso del territorio, por otra parte, considera que el incremento del sistema productivo, se da con las inversiones que efectúan las empresas junto con los agentes públicos, con el creciente de la comunidad local (Vázquez Barquero, 2000), en el cual cada ciudad o región encaminadas en el desarrollo, se da a través de la trayectoria tecnológica y productiva de las organizaciones (Vázquez Barquero, 1999).

El desarrollo local se resguarda bajo la flexibilidad que hace frente a los procesos de la organización en la producción, a partir del cual, se llevan a cabo los modelos dentro de las organizaciones que logran hacer flexible la producción entre las que se puede mencionar a las redes empresariales que poseen alta tecnología en los 
sistemas locales, por lo que estas formas de organización lleva a la creación de procesos de crecimiento y cambio estructural (Arocena, 1995; Vázquez Barquero, 1999).

\section{Teoría de juegos}

La Teoría de Juegos tiene su origen en la investigación operativa, tratando de indagar y construir las situaciones problemáticas basadas en términos cuantitativos a través de modelos matemáticos para generar soluciones adecuadas a estos problemas. Dentro de los modelos matemáticos más utilizados en la teoría de juegos es el llamado "dilema del prisionero". A partir de éste modelo se manifiesta la interacción entre dos actores en la cual se obtienen incentivos para proceder individualmente y no dentro de una cooperación, con la finalidad de que se visualice claramente que el resultado obtenido es mucho menor que el adquirido con un acuerdo mutuo.

Con base en lo anterior mencionado, es que se lleva a cabo la aplicación de los acuerdos de cooperación, donde se considera primordialmente el interés de cada empresario, ya que es preciso que los socios puedan generar decisiones que finalmente llevaran a obtener resultados no muy adecuados. A partir de ello es que la alianza entra en el dilema del prisionero en el cual se espera que para adquirir un resultado óptimo sea necesario que las empresas cooperen y trabajen solo para su beneficio (Axelrod, 1984; Parkhe, 1993).

El desarrollo de esta teoría puntualiza sobre que la alianza y su comportamiento se enfocan en la existencia de una interdependencia entre los socios, situación en la que desarrolla la vulnerabilidad de alguna de las partes, por el descontrol de una de las partes. Por tal motivo, es necesario que los socios entiendan la importancia de que tanto uno, como el otro necesitan de sus capacidades para avanzar en el cumplimiento de sus objetivos, cabe mencionar que, a pesar de esto, siempre se verá incorporada la incertidumbre sobre el oportunismo que han ocasionado algunos actores en este tipo de relaciones. Sin embargo, es importante mencionar que las empresas intentaran cambiar el rumbo aumentando el nivel de confianza (Parkhe, 1993).

A partir de lo anterior mencionado es que la Teoría de Juegos ha sido dirigida hacia la estrategia de alianzas y los investigadores han tratado de analizar sus estructuras considerando que las mismas, marcan una tendencia a obtener mejores resultados, para disminuir la incertidumbre, incrementar la estabilidad y reducir la probabilidad del oportunismo.

\section{Teoría del milieu innovador}

La teoría del medio innovador inicia con la relación del desarrollo regional y las nuevas tecnologías, las cuales toman en cuenta que el territorio no está dado a lo anterior, más bien por el contrario que crea una dinámica de los milieux o entornos, en donde se puede considerar que a partir de colectividades regionales se genera la probabilidad de suscitar una dinámica local, en donde se pueda acumular 
dentro del mismo territorio, los recursos colectivos que sean indispensables en la dinámica de su sistema tecno-productivo (Perrin, 1990; Maillat, 1995).

Dentro de las características que llevan a cabo la acción o la detonación de los milieux se encuentran (Maillat, 1995; Méndez, 2000):

a) La lógica de interacción, a partir de la cual, se construyen relaciones entre los empresarios, además, de las capacidades que tiene para alcanzar los acuerdos principalmente en la cooperación para la innovación, que se lleva a partir de la creación de redes de innovación, y en la asignación de un espacio de trabajo común.

b) El aprendizaje colectivo que se ve representado por la capacidad de los empresarios por adaptarse con el tiempo a las transformaciones de su entorno a través la innovación, la creación de empresas y la producción de saber-hacer específicos, junto con una avance tecnológica común que beneficia la transmisión del conocimiento y actuación colectiva.

\section{Teoría de la Agencia}

La Teoría de la Agencia es considerada muy poco en el estudio de las alianzas, sin embargo, analiza el conflicto de intereses que se pueden encontrar en los miembros de una organización (Jensen \& Meckling, 1976) y replicar dentro de los acuerdos entre actores de las asociaciones.

Por otra parte, la Teoría de la Agencia aborda desde otra perspectiva el fenómeno cooperativo, dado que la mayoría de las investigaciones argumentan una y otra vez los beneficios que tienen las empresas al cooperar (Contractor \& Lorange, 1988). A su vez, menciona que a partir del incremento en acciones colaborativas por parte de las empresas surgen algún tipo de alicientes por la dificultad en alinear intereses entre los empresarios, dada la separación en la propiedad y control (Reuer y Ragozzino, 2006). Por tal motivo, Reuer y Ragozzino (2006) argumentaron que la cooperación puede partir de una estrategia más por conservar su mercado que por generar ventajas competitivas (Porter \& Fuller, Coalitions and Global Strategy, 1986).

\section{Teoría del Poder del Mercado}

La Teoría del Poder del Mercado considera que las empresas pueden ampliar su poder competitivo garantizando un lugar mejor posicionado en su mercado. A su vez, Porter (1980) menciona que el lugar en donde se encuentra la empresa dentro de la estructura industrial se establece a partir de una estrategia genérica viable y lo más rentable posible. Un acuerdo cooperativo puede ofrecer a todos los socios posibilidades de mejorar la posición que ocupan en su industria.

Esta teoría es la cual algunas empresas utilizan con la finalidad de diferenciar las posibles alianzas ofensivas y defensivas (Hymer, 1972). Se menciona que las 
alianzas ofensivas corresponden a aquellas que en las cuales se llevan a cabo para desarrollar ventajas competitivas y mantener o mejorar su posición empresarial al disminuir la capacidad competitiva de sus competidores a través de la disminución de costos de producción y logísticos. Con respecto a las alianzas defensivas se consideran estas, a partir de que las organizaciones generan barreras de entrada que las mantienen en la misma posición, lo que permite tener estabilidad en el sector y aumentar sus beneficios, cabe mencionar que estas alianzas se utilizan por las empresas consideradas competitivamente más débiles.

Por otra parte, la teoría del poder del mercado argumenta que la cooperación empresarial, es considerada como una estrategia por la cual se puede llegar a los objetivos de una manera más rápida y efectiva. Pero la misma, no involucra la confianza de los empresarios por lo que, los beneficios a largo plazo solo se lograran si y solo si la cooperación continua.

\section{La teoría de la innovación abierta}

La teoría de innovación abierta, se planea a partir de la concepción entre la correlación positiva de la alianza en los factores de I+D+i (Investigación más Desarrollo más innovación) al argumentar la falta de creación de actividades innovadoras en individual, sino que dentro de las acciones colaborativas, es cuando se desarrollan iniciativas positivas en I+D+i. Por lo tanto, es que se vuelve un hecho constante la proyección positiva de la cooperación empresarial en la innovación (Laursen \& Salter, 2006; Santamaría, Nieto, \& Barge-Gil, 2010).

A partir de lo anterior, es que la innovación se ha tornado como la consecuencia del involucramiento de empresarios que deciden realizar colaboración (Coombs, Harvey, \& Tether, 2003), además, Navarro Arancegui (2002) deduce que la innovación ya no se puede entender como una acción empresarial individual, sino por el contrario, por lo que con respecto a ello, los estudios realizados muestran empresas dentro de los acuerdos colaborativos con otras empresas, son aquellas que han obtenido un mayor incremento en acciones innovadoras (Christensen, Rogaczewska, \& Vinding, 1999). A su vez, las relaciones cooperativas se han hecho más habituales en los procesos de innovación que realizan las empresas (Piga \& Vivarelli, 2004).

\section{Teoría de los sistemas de innovación}

La teoría de los sistemas de innovación, define a las instituciones y a los empresarios con respecto a su actuar, tanto individual, como colectivo en la generación de creaciones, desarrollo y difusión en los actuales acontecimientos de acciones productivas. Lundvall (1992) plantea, la creación de un sistema nacional de innovación (SNI) considerándolo como: "un sistema social dinámico, caracterizado por una retroalimentación positiva con una tendencia a su propia reproducción, donde la capacidad innovadora está determinada por la interacción virtuosa de los actores que conforman el sistema". 
A su vez, también se proyecta un Sistemas Regionales de Innovación (SRI), a partir del cual establecen que las PYMES dan un mayor peso al entorno innovador en los acuerdos de cooperación empresarial, además, encuentran elementos que utilizan como estrategias dinámicas en la economía mundial actual (Albizu, Olazaran, Otero, \& Lavía, 2011). Lo que lleva a plantear la innovación dentro de los procesos colaborativos, interactivos y acumulativos, fundamentados en los conocimientos e interacciones basadas en la confianza.

\section{Teoría de los clúster}

La teoría de los clúster, se basa en concentraciones geográficas naturales de empresas interconectadas, que suministran y proveen a otras organizaciones afines e instituciones conexas que compiten pero que también cooperan (Porter, 1998).

El autor de esta teoría es Michael Porter quien introduce la proximidad, las relaciones de suministro e intercambios tecnológicos, la interacción personal de los empresarios y los lazos sociales que originan la confianza como factores de cooperación, que permite la comunicación efectiva e intercambio de la información al interior de los agrupamientos. Con respecto a la competencia y la colaboración, se argumenta, que ambas pueden cohabitar, debido a que se realizan en distintas dimensiones y diferentes actores, por lo que, la cooperación en ciertos momentos puede apoyar a establecer una competencia adecuada (Porter, 1998). Por otra parte, también, se menciona que las reiteradas relaciones y los acuerdos informales, inician de la constante convivencia y cooperación en una zona geográfica conexa, lo que lleva a impulsar la confianza y la comunicación efectiva.

\section{Teoría de la competitividad sistémica}

La teoría de la competitividad sistemática (Esser, Hillebrand, Messner, \& Meyer Stamer, 1996) señala, que el aprendizaje y la innovaciones son dos factores que van juntos, para la generación de redes de cooperativas en las micro empresas.

Por otra parte, establece que la competitividad sistémica se basa en un fenómeno que intenta proveer un ambiente que conduzca la búsqueda de capacidades competitivas de las empresas, donde el Estado realiza acciones fundamentales.

\section{Teoría de la organización}

La Teoría de la Organización puntualiza, que los acuerdos de cooperación son los medios a través de los cuales se puede gestionar los recursos de la organización, además, de los que pertenecen otras, a su vez, la cooperación lleva a tener accesos a ellos. También, se considera como la estrategia a través de la cual se establecen los vínculos y la relaciones como practicas estratégicas empresariales (Fernandes da Silva, 2005; Martínez, 2001; Medina, Ortiz, \& Sacristán, 2007). Sin embargo, las organizaciones tienen que elegir entre la estrategia de cooperación 0 la estrategia de competencia (Blanchot, 1999; Moronto Sánchez, 2005). 
Investigaciones realizadas por Briones, Laborda y López (2007), Martín del Peso (2004) y Menguzzato (1992), argumentan, que la estrategia de cooperación lleva a mejorar de la competitividad a partir de la necesidad empresarial, además, de lograr la adaptación y las condiciones del entorno, que lleva a la internacionalización de las empresas. En este contexto, Harris y Wheeler (2005) indican, que la generación de vínculos empresariales puede suministra conocimientos en la introducción de nuevos mercados.

\section{Teoría de redes y estructura social}

La teoría de redes y estructura social, se lleva a cabo con la necesidad de explicar de alguna manera más especifica el fenómeno de los facilitadores de la cooperación interempresarial, de acuerdo a lo mencionado por Saxton (1997), donde se propone que la alianza y su comportamiento depende del valor económico asignado a los recursos. Tomando en cuenta, lo anterior mencionado, se puede argumentar que la teoría de redes sociales señala, que a partir del proceder de los empresarios, los objetivos pueden verse afectados dependiendo de la posición establecida en las redes interorganizacionales. Con respecto a Eisenhardt y Schoonhoven (1996), argumentan, que a partir de la necesidad de tener cooperación estratégica y social es que los empresarios deciden establecer una alianza, en donde primeramente se requiere, dado que se consideran vulnerables frente a sus competidores, debido a sus carencias en recursos y en segundo término es para obtener oportunidades de negocio fundamentadas en relaciones, confianza y compromiso (Staveren y Knorringa, 2007).

\section{Teoría del comportamiento estratégico}

La teoría del comportamiento estratégico establece que las empresas aplican los acuerdos cooperativos con la finalidad de poder incrementar los beneficios a largo plazo a partir de mejorar su posición competitiva. A partir de ello, se considera que las empresas establecen alianzas para alcanzar objetivos estratégicos. Por tal motivo, es que la cooperación es apreciada como la posibilidad con la que las empresas pueden posicionarse competitivamente en el mercado (Kogut, 1988). Harrigan (1985) razona sobre las empresas que han acogido las alianzas como una estrategia en los constantes cambios del mercado.

Por otra parte, García Canal (1994) establece a la cooperación interempresarial como una herramienta estratégica, la cual es necesaria alcanzar de dos maneras: primeramente como la oportunidad de conseguir capacidades y conocimientos que las empresas no que la empresa no tienen y en segundo término, espera que los acuerdos cooperativos tengan como objetivo principal realizar actividades que desarrollen las cadenas de valor.

\section{Teoría de la dependencia de recursos}

La Teoría de la Dependencia de Recursos parte del supuesto, que ninguna empresa tiene la capacidad de generar los suficientes recursos de acuerdo a sus necesidades. 
Dicho de otra manera, que las organizaciones no han logrado ser autosuficientes ni para poder suministrarse de la totalidad de recursos mínimos indispensables para su funcionamiento (Montoro Sánchez, 1999). Sin embargo es necesario que las empresas mantengan interacciones con su medio que le permitan conservar su interdependencia (Barley, Freeman, \& Hybels, 1992).

La dependencia de recursos con la que pueden llegar a contar las organizaciones en algún momento lo llevara a tolerar las limitaciones interpuestas por otras organizaciones. Con referencia a lo anterior, es que se consideran como un factor primordial para las organizaciones en sus acciones interorganizativas, en el cual se comprenden como las capacidades de influenciar a otros para que se lleve a cabo la interacción cooperativa. Por tal motivo, es que las empresas pueden ser visualizadas como grandes grupos de interés, en donde siempre se podrá adquirir algo a su favor 0 en caso contrario confrontaciones de intereses, preferencias y objetivos (Pfeffer \& Nowak, 1976).

Dentro de la teoría de dependencia de recursos, se argumenta, que las alianzas estratégicas aparecen como alternativas cuando se encuentran con la necesidad realizar acuerdos basados en los intereses y objetivos de las organizaciones al no contar con los recursos suficientes para su desarrollo, además, la alta dependencia genera dificultad en la gestión de las mismas y los posiciona en desventaja frente a sus colaboradores (Montoro Sánchez, 1999). Por otra parte, será conveniente llegar a un equilibrio en necesidad de autonomía y reducción de incertidumbre, para poder utilizar el mercado o cooperar con otras organizaciones (Oliver, 1990).

\section{Teoría de la economía industrial}

La teoría de la economía Industrial, lleva a analizar las alianzas estratégicas mediante los acuerdos en donde se puede llegar a conseguir una mejor posición de mercado para lograr intervenir en las decisiones de precio, calidad y naturaleza de los productos.

Los acuerdos de cooperación, se constituyen entre los socios para lograr conseguir la disminución en rivalidad y garantizar un posicionamiento de mercado conjuntamente.

Ho Park y Zhou (2005) exponen, que se han estado desarrollando demasiadas alianzas entre competidores, a lo que argumentan, que su principal objetivo es el obtener una mejor posición en los mercados y no solo el de obtener beneficios, además, de que estas alianzas se llevan a cabo no para acceder a beneficios inmediatos sino más bien dicho para impedir futuras pérdidas. 


\section{TEMPORALIDAD DE LAS TEÓRIAS ORGANIZACIONALES EN LA COOPERACION EMPRESARIAL}

Las teorías planteadas en la presente investigación, tienen como objetivo analizar todos aquellos momentos y situaciones por medio de los cuales, distintos teóricos decidieron argumentar sobre la importancia de las acciones colaborativas en el ámbito empresarial, con la finalidad de que este tipo de acciones tengan un sustento teórico para establecer estrategias necesarias e implementarlas dentro las organizaciones. Cabe mencionar que esto se basa en las necesidades fehacientes como: crecimiento empresarial, desarrollo de habilidades, adquisición de conocimientos, generación de innovación, adquisición de tecnología, posicionamiento de mercado e incremento en su competitividad.

El establecer una temporalidad de las teorías organizacionales, permite examinar las aquellas que a través del tiempo han consideradas de un momento a otro para la generación de cooperación entre las organizaciones como una posible solución a las problemáticas planteadas en los contextos en que se llevaron a cabo, además, parten del supuesto en que la aplicación de un sistema cooperativo pueda ayudar a las empresas a resolver o mejorar sus circunstancias económicas, productivas y sociales.

\section{Línea de tiempo de teorías organizacionales}

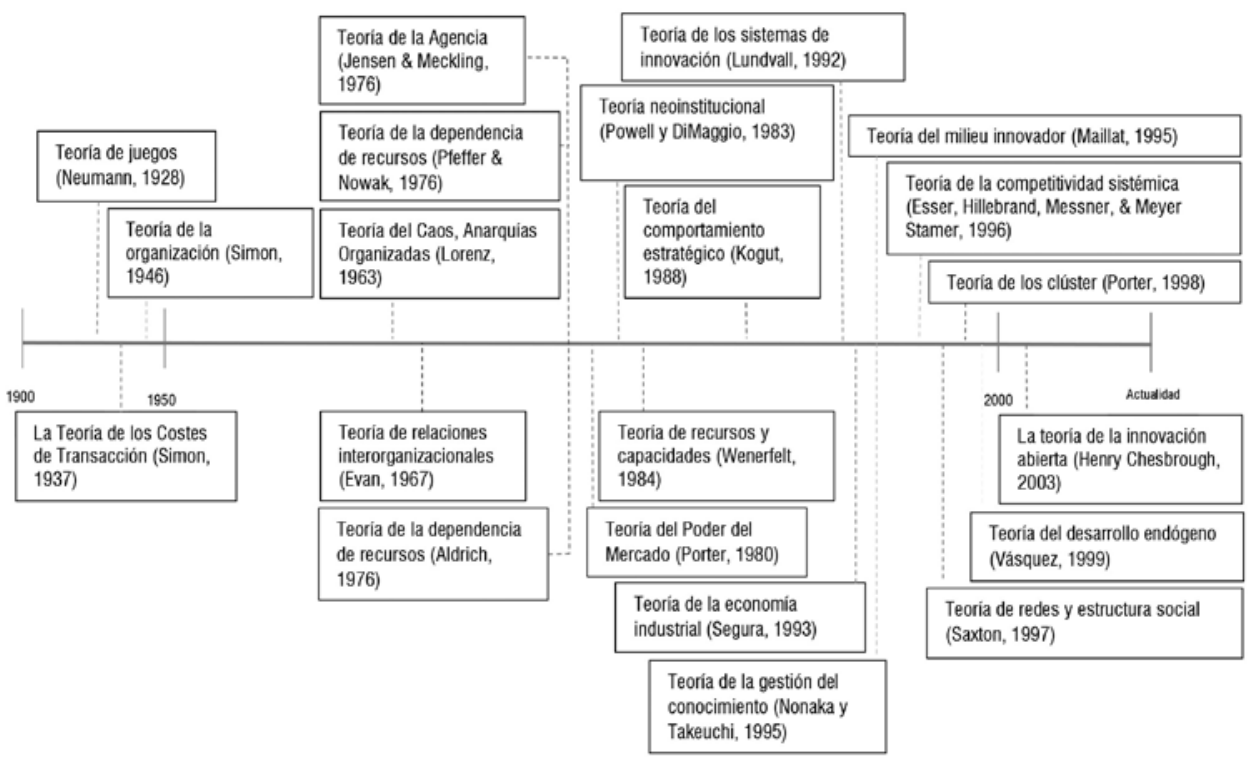

Fuente: Elaboración propia

Visualizando la anterior línea de tiempo, sobre las teorías organizacionales, se puede mencionar que cada una de estas teorías introduce la necesidad del trabajo colaborativo y se puede establecer como la estrategia necesaria para que las 
organizaciones empresariales con las carencias que cada teoría refiere, tenga que aplicar la cooperación interempresarial con la finalidad de sustentar las condiciones que les acontece en su entorno institucional.

El hecho que desde 1928 los teóricos y las organizaciones hablen de la importancia de la cooperación empresarial, permite puntualizar que las acciones colaborativas dentro del contexto empresarial se pueden considerar de una u otra manera, como estrategias para abordar desde distintas áreas del conocimiento las problemáticas que están presentes en su entorno.

\section{METODOLOGÍA}

La metodología utilizada para el desarrollo de la presente investigación, parte de la revisión documental, que lleva a la exploración exhaustiva de la información y búsqueda bibliográfica como: libros, artículos, periódicos, entre otros, para lograr obtener lo que tenga mayor relevancia, con objetivo el analizar y correlacionar las distintas teorías que abordan la cooperación empresarial, para generar una perspectiva simplificada del estudio de los distintos aportes sobre la temática (Hernández, Fernández, \& Baptista, 2010).

\section{DISCUSIÓN}

El establecer una línea de tiempo nos permite visualizar desde una mejor perspectiva las teorías organizativas a través de las cuales se ha visto tratada la cooperación empresarial como una acción empresarial para contrarrestar muchos de los problemas que se han presentado en algunos de los sectores más vulnerables por la globalización y la apertura de fronteras a productos de menor calidad, además, el hecho de concebir que desde hace casi un siglo se viene tratando este tema sin tener una aportación con referente al aporte que tiene la cooperación empresarial, permite discurrir sobre la eficacia de la acción sobre las organizaciones.

La cooperación empresarial como se ve en la línea de tiempo, ha sido tratada en muchas de las teorías organizacionales, sin embargo, es relevante el encontrar hacia donde van dirigidas cada una de estas teorías y establecer una directriz de acciones para los cuales se establece esta herramienta como le llaman algunos teóricos, porque si bien se argumenta, que es una sirve para contrarrestar los ataques constantes de las grandes empresas hacia las pequeñas, además, de encontrar que tan importante es el que se considere parte de la tareas para establecer una mejora en la competitividad de las organizaciones.

Dentro del estudio de las teorías se encontró que la discrepancia generada por algunos teóricos sobre el argumento de que la cooperación empresarial se aplicaba dentro de las organizaciones para la mejora competitiva de las mismas, nos llevó a 
realizar una interacción de las variables donde de acuerdo a la imagen 2, nos llevó a localizar las siguientes interacciones:

\section{Tabla 1. Interacción de cooperación empresarial con teorías organizativas}

\begin{tabular}{|l|l|}
\hline Acción empresarial & Teorías aplicadas \\
\hline Gestión del & $\begin{array}{l}\text { Teoría de la organización (Simon, 1946); Teoría de relaciones } \\
\text { interorganizacionales (Evan, 1967); Teoría de la dependencia de } \\
\text { recursos (Pfeffer \& Nowak, 1976); Teoría neoinstitucional (Powell } \\
\text { y DiMaggio, 1983); Teoría de recursos y capacidades (Wenerfelt, } \\
\text { 1984); Teoría del comportamiento estratégico (Kogut, 1988); Teoría } \\
\text { de los sistemas de innovación (Lundvall, 1992); Teoría de la gestión } \\
\text { del conocimiento (Nonaka y Takeuchi, 1995); Teoría del milieu } \\
\text { innovador (Maillat, 1995). }\end{array}$ \\
\hline $\begin{array}{l}\text { Habilidades } \\
\text { directivas }\end{array}$ & $\begin{array}{l}\text { La Teoría de los Costes de Transacción (Simon, 1937); Teoría del } \\
\text { Caos, Anarquías Organizadas (Lorenz, 1963). }\end{array}$ \\
\hline recursos & $\begin{array}{l}\text { La Teoría de los Costes de Transacción (Simon, 1937); Teoría de la } \\
\text { organización (Simon, 1946); Teoría de la dependencia de recursos } \\
\text { (Pfeffer \& Nowak, 1976); Teoría de la dependencia de recursos } \\
\text { (Aldrich, 1976); Teoría de recursos y capacidades (Wenerfelt, 1984); } \\
\text { Teoría de redes y estructura social (Saxton, 1997). }\end{array}$ \\
\hline $\begin{array}{l}\text { Mejora en } \\
\text { procesos } \\
\text { productivos }\end{array}$ & $\begin{array}{l}\text { La Teoría de los Costes de Transacción (Simon, 1937); Teoría del } \\
\text { Poder del Mercado (Porter, 1980); Teoría del comportamiento } \\
\text { estratégico (Kogut, 1988); Teoría de Ios sistemas de innovación } \\
\text { (Lundvall, 1992); Teoría del milieu innovador (Maillat, 1995); Teoría } \\
\text { del desarrollo endógeno (Vásquez, 1999). }\end{array}$ \\
\hline Innovación \\
y desarrollo \\
tecnológico
\end{tabular}

Fuente: Elaboración propia.

El establecer una correlación entre las teorías organizativas y la cooperación empresarial permite partir del hecho de que las afectaciones empresariales hablando de gestión del conocimiento, comercialización, innovación y desarrollo tecnológico, etc. interactúan entre sí, como acciones en conjunto que potencializan 
las capacidades de las pequeñas organizaciones, y que a través de estas capacidades pueden generar un sinergia para competir frente a sus grandes competidores y lograr sobrevivir a la globalización que les consume su mercado y margina sus productos, por no poder competir contra ellos.

\section{CONCLUSIONES}

Las distintas teorías parten de una necesidad para generar sustentabilidad empresarial, considerando lo anterior, se puede encontrar que la sinergia de ciertas acciones o estrategias abonan al desarrollo y crecimiento de las organizaciones, tal como lo mencionan en las Teorías Organizacionales estudiadas en el presente documento.

Una parte importante a recalcar a partir del análisis de estas teorías, es el enfoque que cada una de ellas tiene hacia las acciones de cooperación y la implementación de las mismas, por tal motivo, es importante argumentar que dentro de los distintos factores que influyen en las organizaciones que implementan la cooperatividad, se realiza a partir de una necesidad para gestionar un cambio que permita tener una mejor posición en el mundo empresarial.

Dentro de las acciones aplicadas, se pueden destacar aquellas que aparecen en la imagen 2, que corresponde a las teorías que se enfocan principalmente en el desarrollo de una ventaja competitiva con la cual los autores consideran que se pueden alcanzar a partir de la cooperación empresarial. 


\section{Imagen 2}

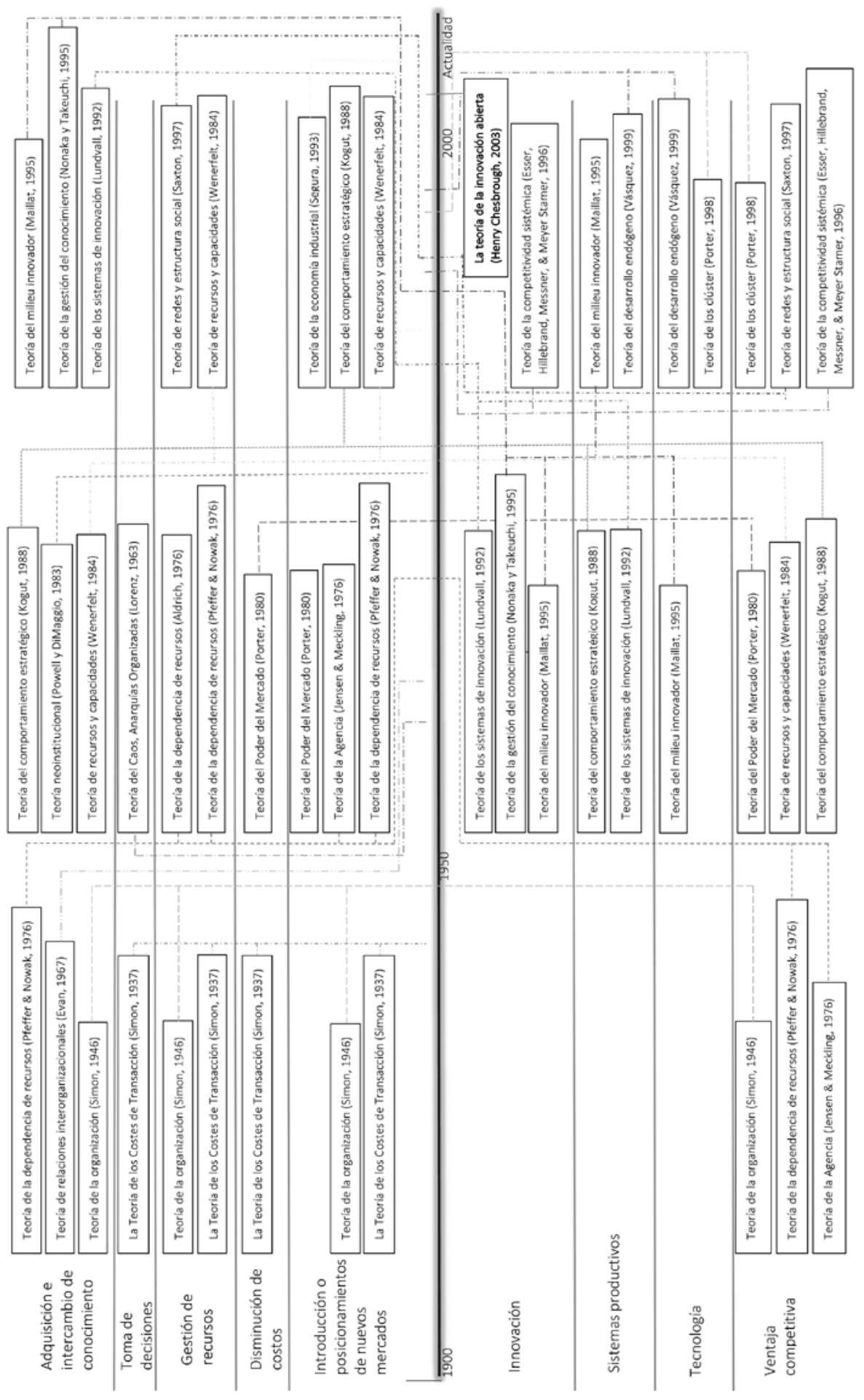

Fuente: Elaboración propia. 
Analizando las imagen 2, se puede argumentar que las teorías organizacionales que consideran la cooperación empresarial dentro de sus principios estratégicos, se dividen de igual manera: en el abordaje que cada una de estas teorías tienen; en el involucramiento de las acciones colaborativas como es: adquisición e intercambio de conocimiento, toma de decisiones, gestión de recursos, disminución de costos, introducción o posicionamiento de nuevos mercados, innovación, sistemas productivos, tecnología y ventajas competitivas.

A partir de lo anterior, se puede considerar, que las teorías organizacionales abonan a la cooperación empresarial en las distintas estrategias que pueden generar dentro de las organizaciones, un cambio que permita poder colocarse dentro del mundo empresarial como empresas competitivas, dado, que uno de sus principales objetivos, es el desarrollo y posicionamiento de las compañías frente a otras.

Por tal motivo, se concluye con que el estudio de las teorías organizacionales, su análisis en la temporalidad y la observación a partir de las acciones en las cuales se podría influir dentro de las empresas, permite a quienes estudian la cooperación empresarial tener un contexto amplio de las situaciones estudiadas con anterioridad para generar con mayor facilidad su abordaje.

\section{REFERENCIAS}

ALBIZU, E., Olazaran, M., Otero, B., \& Lavía, C. (2011). Innovación en las pymes industriales: una visión desde el modelo interactivo. Revista Internacional de Organizaciones, 7, 17-43.

ALDRICH, H. (1976). Resource dependence and interorganizational relations: relations between local employment service offices and social service sector organizations. Administration and Society, 419 - 454.

ALDRICH, H., \& Pfeffer, J. (1976). Environments of organizations. Annual Review of Sociology, 79-105.

AROCENA, J. (1995). El Desarrollo Local: Un Desafío Contemporáneo. Caracas, Venezuela: Editorial Nueva Sociedad.

AXELROD, R. (1984). The evolution of cooperation. New York: Basic Books.

BARLEY, S. R., Freeman, J., \& Hybels, R. (1992). Strategic alliances in commercial biotechnology. Harvard Business School Press, NA.

BEJARANO Lugo, B. Y. (2016). Análisis de las Teorías Organizacionales por la metáfora del organismo y la Evolución de Lamarck. Revista Científica de FAREM-Estelí, 82 - 91. 
BLANCHOT, F. (1999). Les accords inter-firmes et concepts associés: une grille de lecture en terme d'interpénétration organisationnelle. Presses Universitaires de Nancy, 328.

BRIONES, A., Laborda, F., \& López, C. (2007). Visión estratégica y praxis de la cooperación empresarial. FISEC-Estrategias, 8(6), 86-107.

CABANELAS, J., \& Sáez, D. (1997). Cooperar para competir con éxito. Pirámide, 148.

CASANI, F. (1996). La naturaleza de la cooperación empresarial: delimitación del concepto y principales enfoques teóricos. Dirección y Organización, 17(1), $67-77$.

CHRISTENSEN, J. L., Rogaczewska, A. P., \& Vinding, A. L. (1999). Summary report of the Focus Group on Innovative Firms Network. OECD, NA.

COLLINS, D., \& Montgomery, C. (1995). Competing on resources: Strategy in the 1990s. Harvard Business Review, 73(1), 119.

COMISIÓN Europea. (2015). La UE incrementa la inversión y la cooperación empresarial con América. Comisión Europea - Comunicado de prensa.

CONTRACTOR, F. J., \& Lorange, P. (1988). Why Should Firms Cooperate? The Strategy and Economics Basis for Cooperative Ventures. New Lexington Press, NA.

COOMBS, R., Harvey, M., \& Tether, B. S. (2003). Analysing distributed processes of provision and innovation. Industrial and Corporate Change, 11251155.

DAS, T. K., \& Teng, B.-S. (2000). A resource-based theory of strategic alliances. Journal of Management, 26(1), 31 - 61.

DOLÁN, S., García, S., \& Auerbach, A. (2003). Understanding and Managing Chaos in Organisations. International Journal of Management, 20(1), 23.

EISENHARDT, K. M., \& Scoonhoven, C. (1996). Resource-based view of strategic alliances formation: strategic and social effects in entrepreneurial firms. Organization Science, 7(2), 136.

EMERSON, R. M. (1962). Power-Dependence Relations. American Sociological Review, 27, 31 - 41.

ESCRIBÁ, A., \& Menguzzato, M. (1999). Determinantes y Efectos del Comportamiento Cooperativo de los Socios en las Alianzas entre Empresas. Revista ICADE, 48, 25 - 43. 
ESSER, K., Hillebrand, W., Messner, D., \& Meyer Stamer, J. (1996). Competitividad Sistémica: nuevo desafío par a las empresas y la política. CEPAL(59), NA.

EVAN, W. M. (1967). La órbita de la organización: Hacia una teoría de las relaciones interorganizacionales. OMEB, NA.

FERNANDES da Silva, T. (2005). La cooperación interempresarial: nuevas estrategias empresariales para pequeñas empresas en el proceso de desarrollo local. Pasos, 3(1), 125 - 141.

GARCIA Canal, E. (1994). Tendencias empíricas en la conclusión de acuerdos de cooperación. Esic Market, 115 - 129.

GULATI, R., \& Westphal, J. D. (1999). Cooperative or Controlling? The Effects of CEOBoard Relations and the Content of Interlocks on the Formation of Joint Ventures. Administrative Science Quarterly, 44(3), 473-506.

HARRIGAN, K. R. (1985). Strategies for Joint Ventures. Lexington: Lexington Books.

HARRIS, S., \& Wheeler, C. (2005). Entrepreneurs' relationships for internationalization: Functions, origins and strategies. International Business Review, 187 - 207.

HERNÁNDEZ, R., Fernández, C., \& Baptista, M. (2010). Metodología de la investigación. México: McGraw Hill.

HO-PARK, S., \& Zhou, D. (2005). Firm heterogeneity and competitive dynamics in alliance formation. Academy of Management Review, 30(3), 531-554.

HYMER, S. H. (1972). The Multinational Corporation and the Law of Uneven Development. Economics and World Order, NA.

INKPEN, A. C., \& Beamish, P. W. (1997). Knowledge, Bargaining Power, and the Instability of International Joint Ventures. Academy of Management Review, 22(1), 177 - 202.

JENSEN, M. C., \& Meckling, W. H. (1976). Theory of the Firm: Managerial Behavior, Agency Costs, and Capital Structure. Journal of Financial Economics, 3, $305-360$.

KOGUT, B. (1988). Joint-ventures theorecal and empirical perspectives. Strategic Management Journal, 319 - 322.

LAURSEN, K., \& Salter, A. (2006). Open for innovation: The role of openness in explaining innovation performance among U.K. manufacturing firms. Strategic Management Journal, 131150. 
LUNDVALL, B. (1992). National system of innovation. Towards a theory of innovation and interactive learning. Pinter Publishers, NA.

LUO, Y. (2002). Contract, cooperation, and performance in international joint ventures. Strategic Management Journal, 23, 903-919.

MAILLAT, D. (1995). Territorial dynamic, innovative milieus and regional policy. Entrepreneurship and Regional Development, 157 - 165.

MARSHALL, A. (1930). Principles of Economics. Londres, Inglaterra: McMillan press.

MARTÍN DEL PESO, M. (2004). Los consorcios monosectoriales de promoción en el proceso de internacionalización de la empresa: Factores de éxito de la cooperación. Recuperado el 15 de 09 de 2018, de Tesis doctoral no publicada, Universidad Rey Juan Carlos, España.: http://www. cervantesvirtual.com/FichaObra.html?Ref $=13122$

MARTínEZ, S. (2001). Aproximación teórica a los acuerdos de cooperación empresarial. Obtenido de (Documento de trabajo sin publicar del programa doctoral Administración y Dirección de Empresas): http://biblioteca.uca. es/sbuca/bibcsoc/doctrab/ful01-1.pdf

MEDINA, S., Ortiz, M., \& Sacristán, M. (2007). Los acuerdos de cooperación y los resultados empresariales: una revisión desde la metodología de eventos. Cuadernos de Estudios Empresariales, 75-93.

MÉNDEZ, R. (2000). Procesos de innovación en el territorio: Los medios innovadores. Civitas Ediciones, 23-59.

MENGUZZATO, M. (1992). La cooperación empresarial: análisis de su proceso. Instituto de la Mediana y Pequeña Industria Valenciana.

MONTORO Sánchez, M. (1999). La Participación Española en Alianzas Estratégicas Tecnológicas. Revista Icade, 247 - 265.

MORONTO Sánchez, M. A. (2005). Algunas Razones para la Cooperación en el Sector de Automoción. Economía Industrial(358), 27 - 36.

NAVARRO Arancegui, M. (2002). La cooperación para la innovación en la empresa española desde una perspectiva internacional comparada. Economía Industrial, 4766 .

NONAKA, I., \& Takeuchi, H. (1995). The Knowledge-Creating Company. Oxford University Press, NA. 
OCDE. (2004). Conferencia Estambul. Organización para la Cooperación y el Desarrollo Económicos.

OLIVER, C. (1990). Determinants of interorganizational relationships: integration and future directions. Academy of Management Review, 13, $241-265$.

PARKHE, A. (1993). Strategic alliance structuring: a game theoretic and transaction cost examination of interfirm cooperation. Academy of Management Journal, 36(4), 794 - 829.

PERRIN, J. C. (1990). Organisation industrielle: la composante territoriale. Revue d'Économie Industrielle, 21, 276-303.

PFEFFER, J., \& Nowak, P. (1976). Joint Ventures and interorganizational dependence. Administratvive Science Quarterly, 21, 394 - 418.

PFEFFER, J., \& Salancik, G. (1978). The External Control of Organizations: A Resource Dependence Perspective. Harper \& Row.

PIDAL Gonzalez, M. J. (2009). La teoría del caos en las organizaciones. Cuadernos Unimetanos , 29 - 33.

PIGA, C., \& Vivarelli, M. (2004). Internal and external R\&D: A sample selection approach. Oxford Bulletin of Economics and Statistics, 457482.

PORTER, M. E. (1980). Competitive Strategy: Techniques for Analyzing Industries and Competitors. Free Press, NA.

PORTER, M. E. (1998). Location, clusters and new microeconomic of competition. Business Economics, 33(1), NA.

PORTER, M. E., \& Fuller, M. (1986). Coalitions and Global Strategy. Harvard Business School Press, NA.

POWELL, W., \& DiMaggio, P. (1983). The Iron Cage Revisited: Institutional Isomorphism and Collective Rationality in Organizational Fields. American Sociological Review, 147-160.

REUER, J. J., \& Ragozzino, R. (2006). Agency Hazards and Alliance Portfolios. Strategic Management Journal, 27, 27 - 43.

RODRÍGUEZ, C. M., \& Wilson, D. T. (2002). Relationship Bonding and Trust as a Foundation for Commitment in U.S.-Mexican Strategic Alliances: A Structural Equation Modeling Approach. Journal of International Marketing, 10(4), 53 - 76. 
SANTAMARÍA, L., Nieto, M., \& Barge Gil, A. (2010). The relevance of different open innovations strategies for R\&D performers. Cuadernos de Economía y Dirección de la Empresa, 93114.

SIMON, H. (1937). Comparative Statistics and the Measurement of Efficiency. Sational Municipal Reviéw, 524 - 527.

STAVEREN, I., \& Knorringa, P. (2007). Unpacking social capital in economic development: How social relation matter. Review of Social Economy, 107 -135 .

URRA, J. (1999). Cooperación interempresarial. Algunas puntualizaciones en una aproximación economica al fenomeno. Dirección y Organización, 22, $37-49$.

VÁZQUEZ Barquero, A. (1999). Desarrollo, redes e innovación. Lecciones sobre desarrollo endógeno. Madrid: Pirámide.

VÁZQUEZ Barquero, A. (2000). Desarrollo endógeno y globalización. Revista Eure, XXVI(79), 47-65.

WESTPHAL, J. D., Boivie, S., \& Han, D. (2006). The Strategic Ímpetus for Social Network Ties:Reconstituting Broken CEO Friendship Ties. Strategic Management Journal, 27, 425 - 445.

WHITE, S., \& Siu-Yun, S. (2005). Distinguishing cost of cooperation and control in alliances. Strategic Management Journal, 26, 913.

WILLIAMSON, O. E. (1979). Transaction cost economics: the governance of contractual relations. Journal of Law and Economics, 22(2), NA.

WILLIAMSON, O. E. (1985). The Economic Institution of Capitalism: Firms, Market, Relational Contracting. The Free Press, 61 - 75.

WILLIAMSON, O. E. (1991). Comparative economic organization: the analysis of discrete structural alternatives. Administrative Science Quarterly, 36, 269 - 296. 